\title{
Tim Taylor and Alan Dorin: Rise of the self-replicators-early visions of machines, Al and robots that can reproduce and evolve
}

\author{
Springer International Publishing, Cham, Switzerland, 121 pp, $€ 84.99$ \\ (Softcover), ISBN: 978-3-030-48,233-6
}

\author{
Stefano Nichele $e^{1,2}$
}

Received: 27 January 2021 / Accepted: 28 January 2021 / Published online: 15 February 2021

(c) The Author(s) 2021

Artificial Life (ALife) aims at synthesizing principles from living systems in order to build artificial systems that show lifelike behaviors. The main motivations for doing so are to better understand how biological systems work, and to derive bottom-up approaches for designing artificial living systems, such as artificial intelligences (AIs) and autonomous robots. Nowadays, major breakthroughs in narrow AI such as AlphaGo [1], AlphaFold [2], GPT-3 [3], and DALL-E [4], generated interest in whether and how artificial general intelligence (AGI) may be achieved. One of the most intriguing candidate paths to reach AGI is considered to be an open-ended evolutionary process that generates more-and-more sophisticated AI forms through inheritance with variations, i.e., open-ended evolution [5]. Therefore, the topics reviewed in the book Rise of the Self-Replicators, i.e., self-reproduction and evolution of machines, may become a major scientific focus beyond the current AI-hype.

But when did the basic ideas on self-reproduction and evolution in machines originate? And how have they developed until today? Is it really possible to design robots and machines that can evolve like biological organisms do and self-replicate? And what is at stake if such technology is ever built?

The book Rise of the Self-Replicators by Taylor and Dorin provides an intriguing historical perspective on the origin of the ideas around self-replication and evolution in machines. The journey starts from the early investigations in the seventeenth and eighteenth century on the relationships between animals and machines, then continues through theoretical works and physical realizations, relevant scientific and

Stefano Nichele

stenic@oslomet.no

1 OsloMet AI Lab, Department of Computer Science, Oslo Metropolitan University, Oslo, Norway

2 Department of Holistic Systems, Simula Metropolitan Centre for Digital Engineering, Oslo, Norway 
non-scientific literature, and ends with the current ongoing and future research, and a discussion of the ethical and philosophical implications of self-replicating technology for the future of mankind. The book is intended for a wide group of people, ranging from the casual reader interested in the topic, to the more experienced researchers in the disciplines that intersect the fields of artificial intelligence, artificial life, and robotics. In the remainder of this review, I will provide a brief summary of each chapter together with my personal interpretation and critical remarks. I will conclude with an overview of strengths and weaknesses of the book.

In Chapter 1, the authors highlight the idea that most of the modern gadgets, e.g., our mobile phones, are entirely manufactured by other machines, i.e., "machines making machines". However, harvesting and transportation of raw materials are not completely automated yet. Could the entire manufacturing process be fully automated in the future? Taylor and Dorin argue that from a theoretical standpoint, a fully automated manufacturing cycle is completely possible. However, there are still engineering obstacles to overcome. Self-replicating machines are broadly grouped in three categories:

Standard self-replicators, machines that can build perfect copies of themselves;

Evolvable self-replicator, machines that can not only replicate but also evolve by the natural selection of heritable variation, and therefore become "better" than their parents;

Manufacturing self-replicators, machines that can self-reproduce as well as carry out other tasks, such as manufacturing other systems.

Chapter 2 contains in an impressive collection of references, anecdotes, and extracts that allow to track back the origins of the early thinking of machines selfreproduction. In particular, in the early seventeenth century Rene Descartes argued that "animals are machines" and therefore their reproduction is merely a physical process. The combination of two key happenings in the nineteenth century, namely the British Industrial Revolution (machines building machines) and the publication of Darwin's masterpiece On the Origin of Species [6], are presented in Chapter 3. Those events, together with the early idea that animals are machines that can evolve, gave birth to the concept of evolvable self-replicators. Chapter 4 deals primarily with literary works of the early twentieth century, including novels, sci-fi texts, and rather speculative scientific works. One notable example is Rossum's Universal Robots (RUR) [7]. In that period, the discussion of the ideas around self-replicating machines reached different areas of society and was more widespread. However, the envisioned futures often pictured machines taking over the world and extinction of the human race.

During the mid-twentieth century (Chapter 5), the first designs and implementations of self-reproducing machines appeared. The most notable example is perhaps the theoretical work of John von Neumann [8] on logical self-reproduction through a machine that, given description of another machine, would be able to build it or make a copy of the description. By feeding the machine with a tape encoding a description of itself, the machine would self-reproduce. von Neumann's design accounted also for creating offspring machines more complex than the parent machine, e.g., through heritable mutations of the representation tape. The formalism used to implement the machine was a cellular automaton, however before his death von Neumann only 
provided the design of his self-replicator. The first actual implementation on a computer had to wait for another 40 years. This chapter highlights the works of Penrose [9] on hardware self-reproduction using chains of wooden blocks, and offers a new perspective on notable cyberneticians that heavily contributed to the ideas of selfreproducing machines while mostly famous for other works, such as Wiener, Pask, Ashby, and Kolmogorov. However, the most significant contribution of Chapter 5 is the well-deserved recognition to Norwegian-Italian scientist Niels Aall Barricelli, the first to actually carry out experiments on self-reproduction and evolution in computers [10], and therefore making him one of the founding fathers of Artificial Life. Section 5.2.1 (pag. 47) provides a detailed explanation of the brilliant experimental setup designed by Barricelli using cellular automata and artificial evolution, and is highly recommended to the readers of this journal.

Chapter 6 presents the most recent developments until present days. This chapter reviews the important contributions by Langton (organizer of the first artificial life workshop) and Ray [11] (Tierra, populations of computer programs competing for resources), as well as Avida [12] (a software for digital evolution), and Artificial Chemistries [13], up to ongoing work by NASA [14] (physical implementations of maker-replicators for the colonization of the Moon). Most of the works in this chapter are only introduced briefly, and the reader is soon redirected to several references and additional readings materials. I would have appreciated a larger portion of this chapter devoted to follow-up works of von Neumann's original idea, such as the contributions on self-replication by Codd [15] (reducing number of CA states from the 29 of von Neumann to 8), Langton [16] (Langton's loop), Byl [17], Chou and Reggia [18], Tempesti [19], Perrier [20], Sayama [21], and Oros and Nehaniv [22]. I would have also valued a mention to Conway's Game of Life as universal constructor [23].

Chapter 7 concludes the book with the author's reflection on challenges and opportunities, risks, and very much needed discussion on ethical implications of self-replicating and evolving machines. Major attentions are dedicated to self-replicators in the context of the development of artificial intelligence and for the potentially disruptive economical effects if such technology is ever realized in full. This chapter is highly relevant for researchers in the more technical disciplines, as well as those working on AI safety and ethics.

In conclusion, I would like to highlight the great work of Taylor and Dorin in finding and reviewing a rather large corpus of rare historical documents from many sources, including several national libraries in different countries (Scotland, England, Australia, New Zealand, Germany). The early works are reviewed with sensationally high precision, while recent and ongoing work is less detailed (perhaps this was somewhat outside the scope of the book). The research community would benefit from a state-of-the-art review of recent and ongoing work on self-reproducing and evolving machines, as an addition to the book or as a standalone review paper. The book Rise of the Self-Replicators is a great read, and highly recommended to all the readers of the journal and their collaborators. 
Open Access This article is licensed under a Creative Commons Attribution 4.0 International License, which permits use, sharing, adaptation, distribution and reproduction in any medium or format, as long as you give appropriate credit to the original author(s) and the source, provide a link to the Creative Commons licence, and indicate if changes were made. The images or other third party material in this article are included in the article's Creative Commons licence, unless indicated otherwise in a credit line to the material. If material is not included in the article's Creative Commons licence and your intended use is not permitted by statutory regulation or exceeds the permitted use, you will need to obtain permission directly from the copyright holder. To view a copy of this licence, visit http://creativecommons.org/licen ses/by/4.0/.

\section{References}

1. D. Silver et al., Mastering the game of Go with deep neural networks and tree search. Nature 529(7587), 484-489 (2016)

2. A.W. Senior et al., Improved protein structure prediction using potentials from deep learning. Nature 577(7792), 706-710 (2020)

3. Brown, TB, et al. Language models are few-shot learners. arXiv preprint arXiv:2005.14165 (2020).

4. Ramesh, A, et al. DALL·E: Creating Images from Text. https://openai.com/blog/dall-e/online (2021).

5. Stanley, KO, et al. Open-endedness: The last grand challenge you have never heard of. https://www. oreilly.com/radar/open-endedness-the-last-grand-challenge-youve-never-heard-of/ O'Reilly Ideas online (2017).

6. C. Darwin, On the Origin of Species by Means of Natural Selection, or the Preservation of Favored Races in the Struggle for Life (John Murray, London, 1859).

7. K. Čapek, Rossumovi Univerzální Roboti [Rossum's Universal Robots] (Prague, Czech Republic, Aventinum, 1920).

8. von Neumann, J, The theory of self-reproducing automata. University of Illinois Press, Urbana, Ill., Edited by A. W. Burks (1966).

9. L.S. Penrose, Mechanics of self-reproduction. Ann Hum Genet 23(1), 59-72 (1958)

10. N.A. Barricelli, Esempi numerici di processi di evoluzione. Methods 6, 45-68 (1954)

11. T.S. Ray, An approach to the synthesis of life., in Artificial Life II, volume X of SFI Studies in the Sciences of Complexity. ed. by C. Langton, C. Taylor, J. Farmer, S. Rasmussen (Addison Wesley, Boston, 1991), pp. 371-408

12. C. Ofria, O.W. Claus, Avida: a software platform for research in computational evolutionary biology. Artificial life 10(2), 191-229 (2004)

13. McCaskill JS, Polymer chemistry on tape: A computational model for emergent genetics. Technical report, MPI for Biophysical Chemistry (1988).

14. A. Ellery, Are self-replicating machines feasible? J. Spacecr. Rockets 53(2), 317-327 (2016)

15. E.F. Codd, Cellular Automata (Academic Press, New York, 1968).

16. C. Langton, Self-reproduction in cellular automata.". Phys. D. 10(1-2), 135-144 (1984)

17. J. Byl, Self-Reproduction in small cellular automata. Phys. D. 34(1-2), 295-299 (1989)

18. J.A. Reggia, A.L. Steven, C. Hui-Hsien, P. Yun, Simple systems that exhibit self-directed replication. Science 259(5099), 1282-1287 (1993)

19. Tempesti G, A New Self-Reproducing Cellular Automaton Capable of Construction and Computation. in Advances in Artificial Life, Proc. 3rd European Conference on Artificial Life. Granada, Spain: Lecture Notes in Artificial Intelligence, vol. 929, Springer Verlag, Berlin. pp. 555-563 (1995).

20. J.-Y. Perrier, M. Sipper, J. Zahnd, Toward a viable, self-reproducing universal computer. Phys. D. 97(4), 335-352 (1996)

21. Sayama, Hiroki "Toward the Realization of an Evolving Ecosystem on Cellular Automata". in Proceedings of the Fourth International Symposium on Artificial Life and Robotics (AROB 4th '99). Beppu, Oita, Japan. pp. 254-257 (1999).

22. N. Oros, And Nehaniv, Chrystopher "Sexyloop: Self-Reproduction, Evolution and Sex in Cellular Automata” (The First IEEE Symposium on Artificial Life, Hawaii, USA, 2007), pp. 130-138 
23. E.R. Berlekamp, J.H. Conway, R.K. Guy, Winning Ways for Your Mathematical Plays, 2nd edn. (A K Peters Ltd/CRC Press, Boca Raton, 2003).

Publisher's Note Springer Nature remains neutral with regard to jurisdictional claims in published maps and institutional affiliations. 